TRANSACTIONS OF THE

AMERICAN MATHEMATICAL SOCIETY

Volume 350, Number 5, May 1998, Pages 1931-1944

S 0002-9947(98)02149-7

\title{
THE $L_{2}$-LOCALIZATION OF $W(n)$
}

\author{
ROBERT D. THOMPSON
}

\begin{abstract}
In this paper we analyze the localization of $W(n)$, the fiber of the double suspension map $S^{2 n-1} \rightarrow \Omega^{2} S^{2 n+1}$, with respect to $E(2)$. If four cells at the bottom of $D_{p} M^{2 n p-1}$, the $p$ th extended power spectrum of the Moore spectrum, are collapsed to a point, then one obtains a spectrum $C$. Let $Q M^{2 n p-1} \rightarrow Q C$ be the James-Hopf map followed by the collapse map. Then we show that the secondary suspension map $B W(n) \rightarrow Q M^{2 n p-1}$ has a lifting to the fiber of $Q M^{2 n p-1} \rightarrow Q C$ and this lifting is shown to be a $v_{2}$-periodic equivalence, hence an $E(2)$-equivalence.
\end{abstract}

\section{INTRODUCTION}

We begin by recalling the following construction from [24]. Consider the fiber sequence

$$
F \longrightarrow Q S^{2 n+1} \stackrel{j_{p}}{\longrightarrow} Q D_{p} S^{2 n+1}
$$

where $j_{p}$ is the James-Hopf map and $D_{p} S^{2 n+1}$ is the $p^{\text {th }}$ extended power construction on the sphere. The stabilization map $S^{2 n+1} \rightarrow Q S^{2 n+1}$ lifts to a map $S^{2 n+1} \rightarrow F$, and in [24] it is shown that this lifting induces an isomorphism in complex $K$-theory. It follows that there is an equivalence $L_{1} S^{2 n+1} \cong L_{1} F$ where $L_{1}$ stands for Bousfield localization with respect to $K$-theory on the category of spaces. This result enables one to get a handle on $L_{1} S^{2 n+1}$ since the functor $L_{1}$ is reasonably well behaved on fiber sequences, $L_{1}$ of an infinite loop space is something very close to the localization of the corresponding spectrum, and $K$-theory localization stably is well understood.

The aim of this paper is to explore an analogous construction for $L_{2} W(n)$. $L_{2}$ refers to Bousfield localization with respect to the $p$-local homology theory $E(2)$ with coefficients $E(2)_{*}=Z_{(p)}\left[v_{1}, v_{2}, v_{2}^{-1}\right]$ (for example see [28]). $W(n)$ is the homotopy fiber of the double suspension map $S^{2 n-1} \rightarrow \Omega^{2} S^{2 n+1}$, localized at a prime $p$. For technical reasons which probably have to do with our method of proof more than anything else, we will assume $p \geq 5$. The analogue of the stabilization map is a 'secondary suspension map', which is a map $W(n) \rightarrow Q M^{2 n p-2}$ that is degree one on the bottom Moore space. Here $M^{k}$ denotes a mod $p$ Moore space with top cell in dimension $k$. There are various constructions of maps such as this, for example see [8]. It will be more convenient to start with a delooped version of the secondary suspension. In [12] it is shown that there exists a delooping of $W(n)$,

Received by the editors July 23, 1996.

1991 Mathematics Subject Classification. Primary 55P60; Secondary 55Q52, 55T15.

Key words and phrases. $L_{2}$-localization, double suspension, $v_{2}$-periodicity.

The author was partially supported by PSC-CUNY Grant 667399 .

(c)1998 American Mathematical Society 


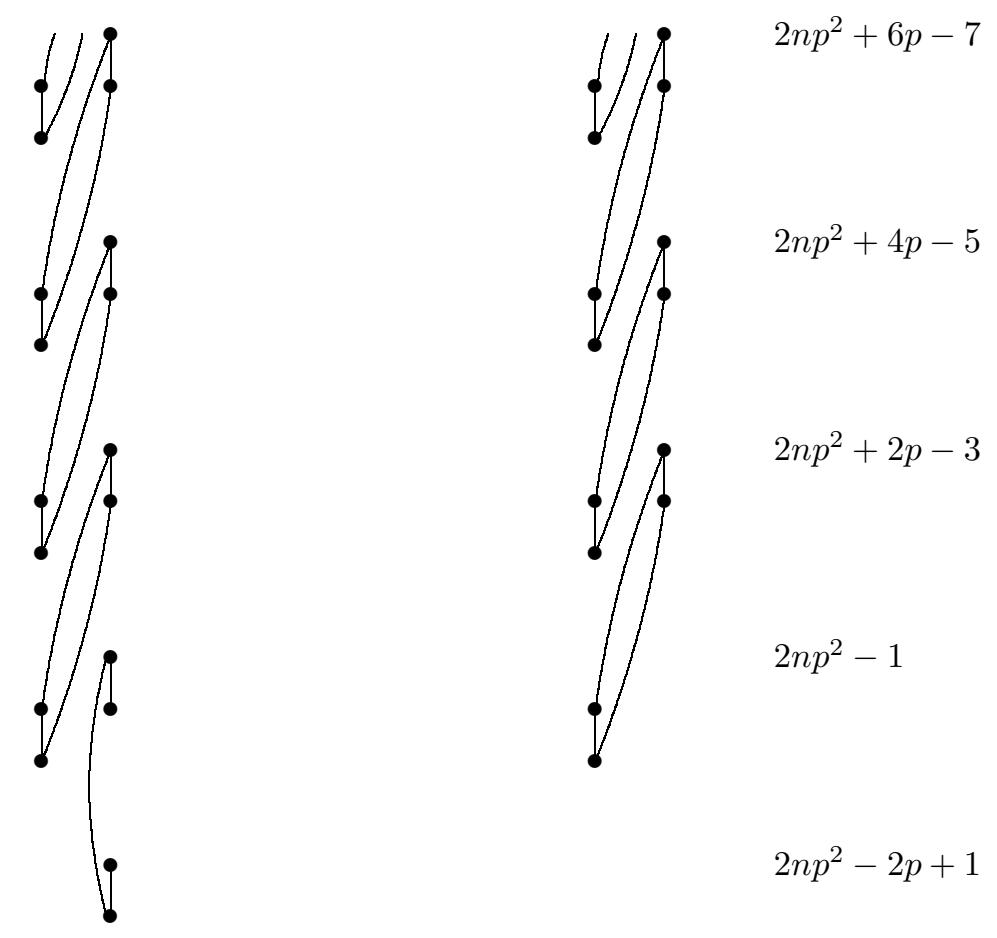

Figure 1. Cell diagram of $D_{p} M^{2 n p-1}$ and $C=D_{p} M^{2 n p-1} / X$

denoted $B W(n)$. It follows from the construction of $B W(n)$ that there is a map $B W(n) \stackrel{\sigma}{\rightarrow} Q M^{2 n p-1}$ which has degree one on the bottom cell. See [12] for details.

Consider the James-Hopf map

$$
Q M^{2 n p-1} \stackrel{j_{p}}{\longrightarrow} Q D_{p} M^{2 n p-1} .
$$

The left side of Figure 1 gives a cell diagram for $D_{p} M^{2 n p-1}$. The short and long lines represent the actions of the Milnor primitives $Q_{0}$ and $Q_{1}$ respectively. Note the four cells near the bottom in dimensions $2 n p^{2}-1,2 n p^{2}-2,2 n p^{2}-2 p+1$, and $2 n p^{2}-2 p$. Denote this 4-cell complex by $X$. Since $p$ is odd, $X$ can be collapsed to a point. Let $C$ denote the complex $D_{p} M^{2 n p-1} / X$ which is pictured on the right side of Figure 1, and consider the fiber sequence

$$
G^{n} \stackrel{i}{\longrightarrow} Q M^{2 n p-1} \longrightarrow Q C,
$$

where the second map is the James-Hopf map $j_{p}$ composed with $Q(\pi)$ where $\pi$ is the collapse map.

In the case of the sphere $S^{2 n+1}$, the lifting of the stabilization map exists for purely dimensional reasons. Since $B W(n)$ is not finite dimensional, a secondary suspension map does not lift for such a simple reason.

Our first result is the following: 
Theorem 1.2. Assume $p \geq 5$ and $n \geq 1$. There exists a map

$$
\sigma_{1}: B W(n) \rightarrow \Omega^{2 p} B W(n+1)
$$

which is degree one on the bottom Moore space. The mapping telescope of the diagram

$$
B W(n) \rightarrow \Omega^{2 p} B W(n+1) \rightarrow \Omega^{4 p} B W(n+2) \rightarrow \ldots
$$

is $Q M^{2 n p-1}$. If we let $\sigma: B W(n) \rightarrow Q M^{2 n p-1}$ denote the inclusion into the telescope, then there exists a map $\lambda: B W(n) \rightarrow G^{n}$ such that $i \circ \lambda=\sigma$.

This will be proved in section 2 by analyzing some properties of the James-Hopf maps. The hypothesis that $p \geq 5$ is required in order to use certain properties of Gray's delooping of $W(n)([12])$.

Our main result is the following:

Theorem 1.3. Assume $p \geq 5$ and $2 n p-2-k$ is sufficiently large. Then

$$
\lambda: \Omega^{k} B W(n) \rightarrow \Omega^{k} G^{n}
$$

induces an isomorphism in $E(2)_{*}$, hence

$$
L_{2} \Omega^{k} B W(n) \simeq L_{2} \Omega^{k} G^{n} .
$$

Just how large $2 n p-2-k$ must be for the theorem to hold is discussed below.

In [24] the $K$-theory isomorphism induced by the map $S^{2 n+1} \rightarrow F$ is established by direct calculation of $K_{*}(F)$ relying on, among other things, the results of [27]. Techniques for calculating the $E(2)$-homology of spaces such as $\Omega^{k} B W(n)$ and $\Omega^{k} G^{n}$ are not in place yet, so Theorem 1.3 will be deduced from Theorem 1.5 stated below, via the following theorem of A. K. Bousfield [3]. In order to state this we recall some definitions.

For each $m \geq 1$, let $V_{m-1}$ denote some finite cell complex which has type $m$, i.e. $K(i)_{*} V_{m-1}=0$ if $i<m$ and $K(m)_{*} V_{m-1} \neq 0$, where $K(i)$ is the $i$ th Morava $K$-theory spectrum (see [28]). Let $v: \Sigma^{d} V_{m-1} \rightarrow V_{m-1}$ be a $v_{m}$ self map, i.e. a map inducing an isomorphism in $K(m)_{*}$ and inducing the zero map in $K(i)_{*}$ if $i \neq m$. Define the homotopy groups of a space $Y$ with coefficients in $V_{m-1}$ by

$$
\pi_{t}\left(Y ; V_{m-1}\right)=\left[\Sigma^{t} V_{m-1}, Y\right]
$$

and define the $v_{m}$-periodic homotopy groups of $Y$, which we will denote by

$$
v_{m}^{-1} \pi_{t}\left(Y ; V_{m-1}\right),
$$

as the colimit of the sequence

$$
\pi_{t}\left(Y ; V_{m-1}\right) \stackrel{v^{*}}{\longrightarrow} \pi_{t+d}\left(Y ; V_{m-1}\right) \stackrel{v^{*}}{\longrightarrow} \ldots
$$

It can be shown that these periodic groups do not depend on the choice of $v$. They do depend on the choice of $V_{m-1}$, however if a map induces an isomorphism in $v_{m}^{-1} \pi_{t}\left(; V_{m-1}\right)$ with one choice of $V_{m-1}$, then it also will with any other choice (Corollary 11.11, [3]). So for purposes of making statements about $v_{m}$-periodic isomorphisms, we are free to choose $V_{n-1}$ as we like.

For each $n$, Bousfield defines an integer $c(n)$. The precise value of $c(n)$ is not known. Very roughly, $c(n)$ is bounded above by the dimension of the bottom cell of a minimally connected type $n$ complex $V_{n-1}$ which is a suspension. Also, $c(n)$ is bounded below by $n+1$. It is known that $c(0)=1$ and $c(1)=2$. Define a 
functor $\tilde{\Omega}$, going from the category of $c(n)$-connected spaces to itself, as the $c(n)$ connected cover of the loop space functor $\Omega$. Let $E_{*}$ be a homology theory. We say a map $f: X \rightarrow Y$ in the homotopy category of $c(n)$-connected spaces is a durable $E_{*}$-equivalence if $\tilde{\Omega}^{k} f: \tilde{\Omega}^{k} X \rightarrow \tilde{\Omega}^{k} Y$ is an $E_{*}$-equivalence for all $k \geq 0$.

The following is distilled from Bousfield [3].

Theorem 1.4 (Bousfield, 13.3 and 13.15 of [3]). Let $f: X \rightarrow Y$ be a map in the homotopy category of $c(n)$-connected spaces. Then $f$ induces an isomorphism in $v_{m}^{-1} \pi_{t}\left(; V_{m-1}\right)$ for all $0 \leq m \leq n$ if and only if $f$ is a durable $E_{*}$-equivalence for all spectra $E$ such that $E^{*}\left(V_{n}\right)=0$.

Such an equivalence is called a $v_{n}$-periodic equivalence. In particular, a $v_{n^{-}}$ periodic equivalence is always an $E(n)_{*}$-isomorphism.

The condition on $n$ and $k$ in Theorem 1.3 can be stated more precisely now: $2 n p-2-k$ is sufficiently large if $\Omega^{k} B W(n)$ is $c(2)$-connected.

Thus by using Bousfield's theorem we see that Theorem 1.3 follows from the following:

Theorem 1.5. Assume that $p \geq 5$ and $n \geq 1$. The map $\lambda: B W(n) \rightarrow G^{n}$ induces an isomorphism in unstable $v_{m}$-periodic homotopy groups for $0 \leq m \leq 2$, i.e $\lambda$ is a $v_{2}$-periodic equivalence.

Theorem 1.5 will be proved in section 3 . The proof is an adaptation to the present situation of the methods employed in [25], [23], [22], and [30]. In particular, Theorem 1.5 could be viewed as an odd primary analogue of the main result [25] which deals with the case $p=2$. However there are two significant differences. The first is that in [25], we do not know if there is a map analogous to $\lambda$ of Theorem 1.2. This means that the statement concerning $v_{2}$-periodic homotopy groups does not obviously translate into a result concerning homological localization. The second is that the lambda algebra calculations of [25] for $p=2$ do not readily carry over to the odd primary case.

We deal with this second point by using the results of B. Gray concerning the odd primary lambda algebra [13] and [14]. Thus Theorem 1.5 is concerned with the application of the machinery of [13] and [14] to the unstable Adams spectral sequence. This was part of the original motivation for studying such subquotients of the lambda algebra. See [21], [15], [22], and [30].

Remark 1.6. If we localize with respect to $K(2)$ instead of $E(2)$ then we can say more. In [10] it is shown that Bousfield localization with respect to the Morava $K$-theory spectrum $K(n)$ preserves fiber sequences which are double loops except possibly in dimensions $n-1, n$, and $n+1$. Combining this with Theorem 1.3 yields the following corollary:

Corollary 1.7. Let $p \geq 5$ and $2 n p-4>c(2)$. Then there is a map from $L_{K(2)} \Omega W(n)$ to the homotopy fiber of

$$
L_{K(2)} Q M^{2 n p-3} \rightarrow L_{K(2)} Q \Sigma^{-2} C
$$

which induces an isomorphism in homotopy groups except possibly in dimensions 1,2, and 3 .

Furthermore, in [2] Bousfield proves that the localization of any infinite loop space $\Omega^{\infty} Z$ with respect to any spectrum $E$ is again an infinite loop space. There is a certain localization functor associated to $E$ on the category of $(-1)$-connected 
spectra, called the $E_{*} \Omega^{\infty}$ localization, and in [2] it is shown that the $E$-localization of the space $\Omega^{\infty} Z$ is $\Omega^{\infty}$ applied to the spectrum $E_{*} \Omega^{\infty} Z$. Thus Corollary 1.7 shows that the homotopy groups of $L_{K(2)} \Omega W(n)$ could in principle be computed from the LES associated to the $K(2)$-localization of (1.1), if one had explicit information about the $K(2)_{*} \Omega^{\infty}$ localization functor on connective spectra.

The author wishes to thank several people for numerous conversations related to these results. Mark Mahowald conjectured Theorem 1.2, and Mahowald and Bill Richter helped with the proof. Brayton Gray spent much time explaining his work to the author and made a number of suggestions which improved the present work.

\section{JAMES-HOPF MAPS}

Theorem 1.2 follows from some basic properties of James-Hopf maps in conjunction with some properties of Gray's construction of $B W(n)$. We recall James-Hopf maps:

For nonnegative integers $k$ and $q$ (or $k$ infinite) and each space $X$ there are James-Hopf maps

$$
j_{q}: \Omega^{k} \Sigma^{k} X \rightarrow Q D_{k, q} X
$$

natural in $X$, where $D_{k, q} X$ is the extended power space $C_{k}(q)^{+} \wedge_{\Sigma_{q}} X^{[q]}$. Here $C_{k}(q)$ is the space of ordered $q$-tuples of little cubes disjointly embedded in $I^{k}$. If $k$ is infinite, we simply write $D_{q} X$. The maps $j_{q}$ are defined in [6]. In [4] an important Cartan formula is proved for the James-Hopf maps, and in [18] various compatibility relations between the James-Hopf maps are established which are extremely useful.

Taking the wedge sum of the adjoints of the James-Hopf maps yields a map of spectra

$$
J: \Sigma^{\infty} \Omega^{k} \Sigma^{k} X \rightarrow \bigvee_{q \geq 1} \Sigma^{\infty} D_{k, q} X
$$

which is a stable equivalence. Such a stable splitting was first established in [17] for $k=\infty$ and [29] for finite $k$ and then generalized in [6] and [4]. Such a splitting is not unique of course. Throughout this paper $j_{q}$ will always refer to the James-Hopf maps of [6], [4], and the stable splitting of $\Omega^{k} \Sigma^{k} X$ will be the one in (2.1) induced by the maps $j_{q}$ unless otherwise noted.

In [12] Gray shows that $W(n)$ is a loop space. More precisely, he shows that there exists a space $B W(n)$, together with a map $\Omega^{2} S^{2 n+1} \stackrel{\nu}{\rightarrow} B W(n)$ such that the homotopy fiber of $\nu$ is $S^{2 n-1}$. For $p$ odd, $B W(n)$ is shown to be an H-space, and for $p \geq 5, \nu$ is an H-map. In what follows we need $\nu$ to be an H-map, hence the hypothesis in Theorem 1.2 that $p \geq 5$. Furthermore, in Proposition 7 of [12], it is shown that there is a splitting

$$
\begin{aligned}
\Sigma^{2} \Omega^{2} S^{2 n+1} & \cong \Sigma^{2}\left(S^{2 n-1} \times B W(n)\right) \\
& \cong \Sigma^{2}\left(S^{2 n-1} \vee B W(n) \vee \Sigma^{2 n-1} B W(n)\right) .
\end{aligned}
$$

In [8], it is shown that the James-Hopf map admits a factorization

$$
\Omega^{2} S^{2 n+1} \rightarrow \Omega^{2 p} \Sigma^{2 p} M^{2 n p-1} \rightarrow Q M^{2 n p-1}=Q D_{2, p}\left(S^{2 n-1}\right) .
$$


Definition 2.3. Let $s: \Sigma^{2} B W(n) \rightarrow \Sigma^{2} \Omega^{2} S^{2 n+1}$ be the right inverse of $\Sigma^{2} \nu$ corresponding to (2.2). Let $\sigma_{1}{ }^{\prime}: B W(n) \rightarrow \Omega^{2 p} \Sigma^{2 p} M^{2 n p-1}$ be the adjoint of the composite

$$
\Sigma^{2 p} B W(n) \stackrel{\Sigma^{2 p-2} s}{\longrightarrow} \Sigma^{2 p} \Omega^{2} S^{2 n+1} \stackrel{\tilde{j}_{p}}{\longrightarrow} \Sigma^{2 p} M^{2 n p-1} .
$$

Finally, let $\sigma_{1}: B W(n) \rightarrow \Omega^{2 p} B W(n+1)$ be the composite

$$
B W(n) \stackrel{\sigma_{1}{ }^{\prime}}{\longrightarrow} \Omega^{2 p} \Sigma^{2 p} M^{2 n p-1} \rightarrow \Omega^{2 p} B W(n+1)
$$

where the second map is $\Omega^{2 p}$ on the inclusion of the bottom cell.

The proof that $Q M^{2 n p-1}$ is the mapping telescope of $\sigma_{1}$ is the same as that in [8]. Note that the map $B W(n) \stackrel{\sigma}{\rightarrow} Q M^{2 n p-1}$ is just

$$
B W(n) \stackrel{\sigma_{1}^{\prime}}{\longrightarrow} \Omega^{2 p} \Sigma^{2 p} M^{2 n p-1} \rightarrow Q M^{2 n p-1}
$$

where the second map is the inclusion.

For the last statement in Theorem 1.2 we need several lemmas.

The following lemma is a variation of Lemma 3.6 of [20]. The difference is that the secondary suspension map $\alpha$ defined in Lemma 3.6 of [20] is not a priori the same as the map $\sigma$ defined here. One can conclude after the fact that $\alpha$ and $\sigma$ are the same since $B W(n)$ splits off of $\Omega^{2} S^{2 n+1}$ stably.

Lemma 2.4. There exists a factorization up to homotopy of the James-Hopf map:

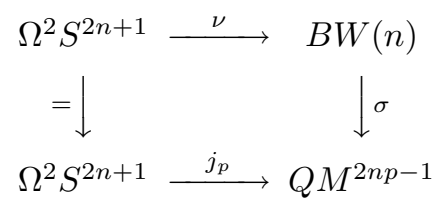

Proof. The mod $p$ homology algebra of $\Omega^{2} S^{2 n+1}$ for $p$ odd is given by ([5])

$$
E\left(\iota, Q_{1} \iota, Q_{1}^{2} \iota, \ldots\right) \otimes P\left(\beta Q_{1} \iota, \beta Q_{1}^{2} \iota, \ldots\right) .
$$

If we assign weights to the monomials by $\operatorname{wt}\left(Q_{1}^{j} \iota\right)=\operatorname{wt}\left(\beta Q_{1}^{j} \iota\right)=p^{j}$ and $\operatorname{wt}(x y)=$ $\mathrm{wt}(x)+\mathrm{wt}(y)$ then the homology of $D_{2, j} S^{2 n-1}$ is the vector space of monomials of weight $j$. It follows that $\Omega^{2} S^{2 n+1}$, localized at $p$, splits stably into a wedge $\bigvee_{j=1}^{\infty} D_{2, j} S^{2 n-1}$ where $j \equiv 0$ or $1(\bmod p)$. Let $J^{-1}$ stand for the homotopy equivalence which is inverse to the stable splitting of (2.1) given by the James-Hopf maps.

It can be verified by an easy calculation in homology that the composite

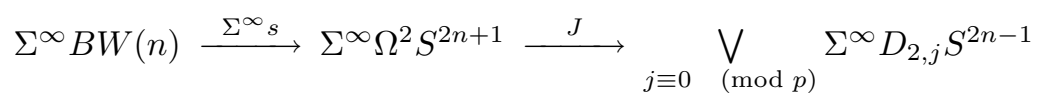

is a homotopy equivalence. Thus we have a stable splitting

$$
\Sigma^{\infty} B W(n) \vee\left(\bigvee_{j \equiv 1} \Sigma_{(\bmod p)} \Sigma^{\infty} D_{2, j} S^{2 n-1}\right) \stackrel{\Sigma^{\infty} s \vee J^{-1}}{\cong} \Sigma^{\infty} \Omega^{2} S^{2 n+1}
$$

Consider the adjoint of the diagram in Lemma 2.4. It is immediate that the adjoint diagram commutes when restricted to the piece $\Sigma^{\infty} B W(n)$. To show that the diagram commutes on the other piece first note that $\tilde{j}_{p}: \Sigma^{\infty} \Omega^{2} S^{2 n+1} \rightarrow$ $\Sigma^{\infty} M^{2 n p-1}$, is null homotopic on the pieces of the splitting where $j \equiv 1(\bmod p)$. Thus the proof of 2.4 is completed by the following lemma. 
Lemma 2.6. The composite map

$$
j \equiv 1 \bigvee_{(\bmod p)} D_{2, j} S^{2 n-1} \stackrel{J^{-1}}{\longrightarrow} \Sigma^{\infty} \Omega^{2} S^{2 n+1} \stackrel{\Sigma^{\infty} \nu}{\longrightarrow} \Sigma^{\infty} B W(n)
$$

is null homotopic.

Proof. This makes use of the Cartan formula for James-Hopf maps given in [4] and the fact that $B W(n)$ is an $\mathrm{H}$-space [12]. There are pairings $D_{k, j} X \wedge D_{k, r} X \rightarrow$ $D_{k, j+r} X$ induced by the inclusion $\Sigma_{j} \times \Sigma_{r} \subset \Sigma_{j+r}$ and the Cartan formula for James-Hopf maps says that these pairings are compatible, via the stable splitting, with the stabilization of the $\mathrm{H}$-space multiplication on $\Omega^{k} \Sigma^{k} X$. In the following diagram we will abbreviate $D_{2, j} S^{2 n-1}$ to $D_{j}$. We will suppress the symbol $\Sigma^{\infty}$ but the diagram is to be understood as being stable.

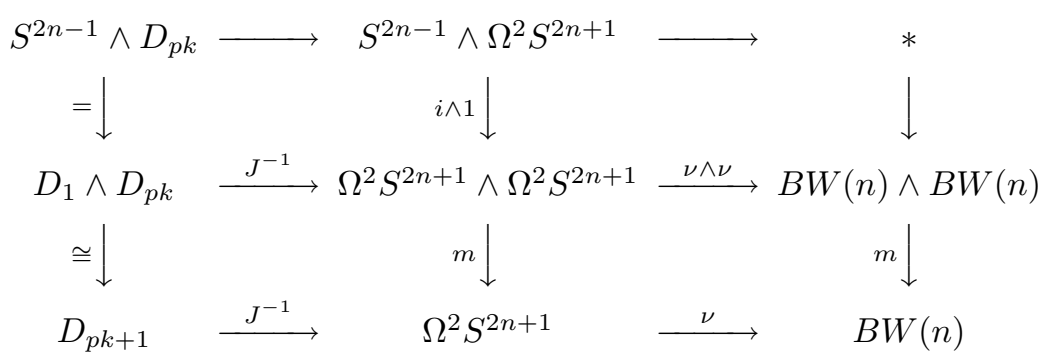

The upper left vertical map is an equivalence because $D_{1}=S^{2 n-1}$. The lower left vertical map induces an isomorphism in homology hence is an equivalence. The lower middle vertical map is the Hopf construction on the $\mathrm{H}$-space multiplication on $\Omega^{2} S^{2 n+1}$. The right hand lower vertical map is the Hopf construction on the $\mathrm{H}$ space multiplication on $B W(n)$. Since $\nu$ is an H-map, the lower right hand square commutes. The upper right square commutes since $S^{2 n-1} \rightarrow \Omega^{2} S^{2 n+1} \stackrel{\nu}{\rightarrow} B W(n)$ is null. This completes the proof of 2.6 .

Before completing the proof of Theorem 1.2 we recall a result from [19] concerning the composite of two James-Hopf maps:

Theorem 2.7 (part of 5.2 of [19]). For $k, n, r, q \geq 1$ let $f_{r, q}^{n}$ be the composite map

$$
\Sigma^{\infty} D_{k, n} X \hookrightarrow \Sigma^{\infty} \Omega^{k} \Sigma^{k} X \stackrel{\Sigma^{\infty} j_{q}}{\longrightarrow} \Sigma^{\infty} Q D_{k, q} X \rightarrow \Sigma^{\infty} D_{r} D_{k, q} X
$$

Then $f_{r, q}^{n}$ is null homotopic if $n>r q$.

Proof. (of Theorem 1.2)

In order to get a lifting $B W(n) \stackrel{\lambda}{\rightarrow} G$ we need to know that the composite $B W(n) \stackrel{\sigma}{\rightarrow} Q M^{2 n p-1} \rightarrow Q C$ is null homotopic. Gray's map $\Omega^{2} S^{2 n+1} \stackrel{\nu}{\rightarrow} B W(n)$ has a right inverse stably (Theorem 8(e) [12]), so by Lemma 2.4 it suffices to show that $\Omega^{2} S^{2 n+1} \stackrel{j_{p}}{\longrightarrow} Q M^{2 n p-1} \rightarrow Q C$ is null homotopic. This is equivalent to a factorization of $j_{p} \circ j_{p}$ through $Q X$, where $X$ is the four cell complex at the bottom of $D_{p} M^{2 n p-1}$ defined in Section 1. See the diagram below. Notice that $X$ is homotopy equivalent to $D_{2, p^{2}}\left(S^{2 n-1}\right)$, so Theorem 1.2 is proved once we know that 
the following square commutes up to homotopy:

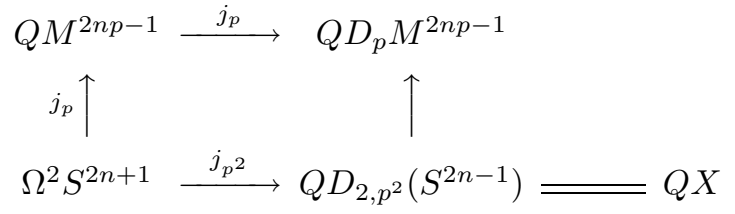

Equivalently, we consider the adjoint diagram and check that it commutes on each piece of the stable splitting of $\Omega^{2} S^{2 n+1}$ :

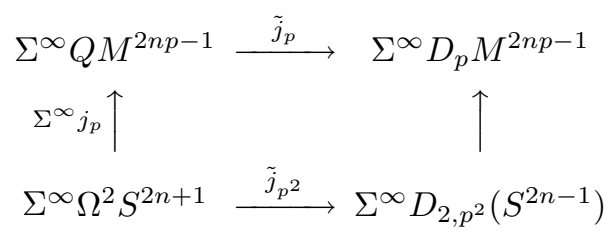

The right hand vertical map is a sort of transfer, defined as the composite

$$
\Sigma^{\infty} D_{2, p^{2}}\left(S^{2 n-1}\right) \hookrightarrow \Sigma^{\infty} \Omega^{2} S^{2 n+1} \stackrel{\Sigma^{\infty} j_{p}}{\longrightarrow} \Sigma^{\infty} Q M^{2 n p-1} \stackrel{\tilde{j}_{p}}{\longrightarrow} \Sigma^{\infty} D_{p} M^{2 n p-1} .
$$

Thus the square (2.8) commutes on the $p^{2}$ piece of the splitting by definition. The bottom horizontal map is null on $\Sigma^{\infty} D_{2, m}\left(S^{2 n-1}\right)$ for each $m \neq p^{2}$. The composite $\tilde{j}_{p} \circ \Sigma^{\infty} j_{p}$ is null on $\Sigma^{\infty} D_{2, m}\left(S^{2 n-1}\right)$ for $m<p^{2}$ for purely dimensional reasons. Finally, $\tilde{j}_{p} \circ \Sigma^{\infty} j_{p}$ is null on $\Sigma^{\infty} D_{2, m}\left(S^{2 n-1}\right)$ for $m>p^{2}$ by Theorem 2.7 .

\section{UNSTABLE $v_{2}$-PERIODIC HOMOTOPY GROUPS}

In this section we will prove Theorem 1.5. To start, we have

Lemma 3.1. $\lambda: B W(n) \rightarrow G^{n}$ induces an isomorphism in $v_{0}^{-1} \pi_{*}(\quad)$ and $v_{1}^{-1} \pi_{*}(\quad)$.

Proof. $v_{0}^{-1} \pi_{*}(\quad)$ is just rational homotopy and both spaces are torsion. The map $\sigma: B W(n) \rightarrow Q M^{2 n p-1}$ induces an isomorphism in $v_{1}^{-1} \pi_{*}(\quad)$ by [30]. To see that $v_{1}^{-1} \pi_{*}(Q C)=0$, use the fact that $C$ has a filtration with subquotients $V(1)$, and so $K(1)_{*} V(1)=0$. By the telescope theorem for $n=1$ (Theorem 4.11 of [1]), we have that stably $v_{1}^{-1} \pi_{*}(V(1))=0$.

We will define unstable $v_{2}$-periodic homotopy groups by taking $V_{1}$ to be the Smith-Toda complex $V(1)$, which we will denote simply by $V$. Since $p \geq 5, V$ has a $v_{2}$-self map $v_{2}: \Sigma^{\left|v_{2}\right|} V \rightarrow V$. Using a $p$-local version of the Freudenthal suspension theorem (see [11]) we see that this $v_{2}$-self map is defined unstably as long as $V$ is at least $d-1$-connected, where $d=\frac{2 p^{2}+1}{p-1}+3$.

Consider the map of pairs

$$
\left(Q M^{2 n p-1}, B W(n)\right) \rightarrow\left(Q M^{2 n p-1}, G^{n}\right) \rightarrow(Q C, *) .
$$

It suffices to show that this induces an isomorphism

$$
v_{2}^{-1} \pi_{*}\left(Q M^{2 n p-1}, B W(n) ; V\right) \stackrel{\cong}{\longrightarrow} v_{2}^{-1} \pi_{*}(Q C ; V) .
$$

The proof of this is based on the modified unstable Adams spectral sequence techniques of [22], [23], [25], [30]. This machinery takes as input certain calculations involving subquotients of the lambda algebra. See [15] and [21]. In the present case, 
the relevant lambda algebra calculations are provided by [13] and [14] so we will use that framework. We recall the construction.

In [13] it is shown that there are spaces $\left\{W_{(0)}^{n}\right\}_{n \geq 0}$ and maps

$$
\Omega W_{(0)}^{2 n-1} \rightarrow \Omega^{3} W_{(0)}^{2 n+1} \rightarrow \Omega^{5} W_{(0)}^{2 n+3} \rightarrow \Omega^{7} W_{(0)}^{2 n+5} \rightarrow \ldots
$$

The two cell complex at the bottom of $\Omega^{2 k+1} W_{(0)}^{2(n+k)-1}$ is $M^{2 n-2}$ and each of the above maps is degree one on this bottom Moore space. The homotopy colimit of this sequence is $Q M^{2 n-2}$. The spaces $W_{(0)}^{2 n-1}$ are defined as follows:

$$
W_{(0)}^{2 n-1}=\operatorname{fiber}\left(\pi_{n}: \Omega^{2} S^{2 n+1} \rightarrow S^{2 n-1}\right)
$$

where

$$
\pi_{n}= \begin{cases}\pi_{n} & \text { from [7] if }(n, p)=1 \\ \phi_{m} & \text { from [12] if } n=p m\end{cases}
$$

Thus $\Omega W_{(0)}^{2 n p-1}=W(n)$.

We need to prove that there is an isomorphism

$$
v_{2}^{-1} \pi_{*}\left(Q M^{2 n p-2}, \Omega W_{(0)}^{2 n p-1} ; V\right) \stackrel{\cong}{\longrightarrow} v_{2}^{-1} \pi_{*}\left(Q \Sigma^{-1} C ; V\right) .
$$

Even though the map $\Omega W_{(0)}^{2 n p-1} \rightarrow Q M^{2 n p-2}$ defined by (3.3) is not necessarily the same as $\Omega \sigma: W(n) \rightarrow Q M^{2 n p-2}$, we will nevertheless see that the proof of (3.5) leads to the proof of (3.2).

In [14] certain subquotients of $\Lambda$, the odd primary lambda algebra, are defined. These are denoted by $\Lambda_{(m)}(n), m \geq-1, n \geq 0$. There are SES's

$$
0 \rightarrow \Lambda_{(m)}(2 n-1) \rightarrow \Lambda_{(m)}(2 n) \rightarrow \Lambda_{(m)}(2 n p-1) \rightarrow 0
$$

and

$$
0 \rightarrow \Lambda_{(m)}(2 n) \rightarrow \Lambda_{(m)}(2 n+1) \rightarrow \Lambda_{(m)}\left(2 n p+2 p^{m+1}-1\right) \rightarrow 0
$$

which yield EHP sequences in homology and a SES

$$
0 \rightarrow \Lambda_{(m)}(2 n-1) \rightarrow \Lambda_{(m)}(2 n+1) \rightarrow \Lambda_{(m+1)}(2 n p-1) \rightarrow 0
$$

which yields the double suspension sequence.

We have

$$
\Lambda_{(m)}=\bigcup_{n=1}^{\infty} \Lambda_{(m)}(n)=E\left(\tau_{0}, \ldots, \tau_{m}\right) \tilde{\otimes} \Lambda
$$

where $E\left(\tau_{0}, \ldots, \tau_{m}\right)$ is the exterior subalgebra of the dual Steenrod algebra $A_{*}$. In those cases where $V(m)$ exists we have $E\left(\tau_{0}, \ldots, \tau_{m}\right)=H_{*} V(m)$ and

$$
H_{*}\left(E\left(\tau_{0}, \ldots, \tau_{m}\right) \tilde{\otimes} \Lambda\right)=\operatorname{Ext}_{A_{*}}\left(H_{*} V(m)\right) .
$$

The chain complex $\Lambda_{(m+1)}(k)$ has a splitting given by the SES's

$$
0 \rightarrow \Lambda_{(m)}(2 n+1) \rightarrow \Lambda_{(m+1)}(2 n+1) \rightarrow \Lambda_{(m)}\left(2 n+2 p^{m+1}+1\right) \rightarrow 0
$$

and

$$
0 \rightarrow \Lambda_{(m)}(2 n+1) \rightarrow \Lambda_{(m+1)}(2 n) \rightarrow \Lambda_{(m)}\left(2 n+2 p^{m+1}-1\right) \rightarrow 0
$$

There are $v_{m}$-self maps

$$
v_{m}: \Lambda_{(m-1)}\left(2 n+2 p^{m}-1\right) \rightarrow \Lambda_{(m-1)}(2 n-1)
$$


and isomorphisms

$$
v_{m}^{-1} \Lambda_{(m-1)}(2 n-1) \cong v_{m}^{-1} \Lambda_{(m-1)}(2 n+1) \cong v_{m}^{-1}\left(E\left(\tau_{0}, \ldots, \tau_{m-1}\right) \tilde{\otimes} \Lambda\right) .
$$

Recall from [22] and [30] that a resolution of a space $X$ is a tower of fibrations,

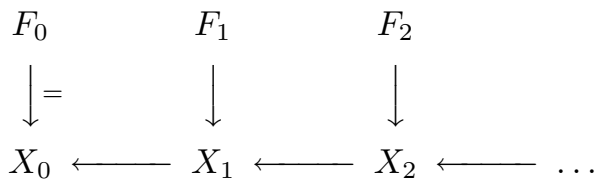

with each fiber $F_{s}$ being a GEM, and compatible maps $f_{s}: X \rightarrow X_{s}$, with $f_{\infty}$ : $X \rightarrow X_{\infty}$ being the $p$-completion. Given a resolution of a space, there is the usual homotopy spectral sequence.

Lemma 3.7. (1) There is a resolution of $W_{(0)}^{2 n-1}$ with

$$
E_{2}^{s, t} \cong H_{*}\left(\Lambda_{(0)}(2 n-1)\right) \text {. }
$$

(2) The map $\Omega W_{(0)}^{2 n-1} \rightarrow \Omega^{3} W_{(0)}^{2 n+1}$ is covered by a map of resolutions, and the induced map of $E_{2}$-terms is $H_{*}\left(\Lambda_{(0)}(2 n-1)\right) \rightarrow H_{*}\left(\Lambda_{(0)}(2 n+1)\right)$ from (3.6).

(3) Let

$$
\Omega^{2} W_{(1)}^{2 n p-1} \rightarrow \Omega W_{(0)}^{2 n-1} \rightarrow \Omega^{3} W_{(0)}^{2 n+1}
$$

be the homotopy fiber sequence of [13]. Then there is a resolution of $\Omega^{2} W_{(1)}^{2 n p-1}$ with $E_{2}^{s, t} \cong H_{*}\left(\Lambda_{(1)}(2 n p-1)\right)$.

Proof. Proposition 6.3 of [25] states that if we are given a map of spaces $f: X \rightarrow$ $Y$, and resolutions of $X$ and $Y$, then there is a map of resolutions covering $f$ if the largest dimensional homotopy class in $\pi_{*} F_{s}$, for the target space $Y$, is in the range through which $f_{s}$, for the source space $X$, is surjective in cohomology. This was used in [22] (Proposition 4.10) and [30] (Theorem 2.27) to produce a map of resolutions covering a secondary suspension map $W(n) \rightarrow \Omega^{2 p} W(n+1)$. The proof of Proposition 6.3 of [25] is the same as the proof of Proposition 4.10 of [22]. If we replace the resolution of the target space $Y$ by the same tower starting in degree $i$, then we have the result that there is a filtration $i$ map of resolutions covering $f$ if the largest dimensional homotopy class in $\pi_{*} F_{s+i}$, for the target, is in the range through which $f_{s}$, for the source, is surjective in cohomology.

We apply this to the map $\pi_{n}: \Omega^{2} S^{2 n+1} \rightarrow S^{2 n-1}$ of (3.4). As usual, take the Adams resolution for $S^{2 n-1}$ with $\Lambda(2 n-1)$ as $E_{1}$-term, and for $\Omega^{2} S^{2 n+1}$ take double loops on the Adams resolution for $S^{2 n+1}$. The map of resolutions needs to be a filtration one map. As in [30], the dimension of a class in $\pi_{*} F_{s+1}$, for $S^{2 n-1}$, is at most

$$
q(n-1)\left[1+p+\cdots+p^{s}\right]+2 n-1 .
$$

This is less than $(2 n-1) p^{s+1}+(p-2) p^{s}$, which is the range through which $f_{s}^{*}$ : $H^{*} \Omega^{2} S^{2 n+1} \leftarrow H^{*} X_{s}$ is onto in the resolution of $\Omega^{2} S^{2 n+1}$.

Proposition 3.3 of [22] (see also 2.20 of [30]) states that if we are given a map of resolutions covering a given map $f$, then there is a resolution of the fiber of $f$, and a long exact sequence of $E_{2}$-terms. It is implicit in [22] that one of the maps in the LES is induced by the map $f$. This last fact is proved explicitly in [23].

For our resolution of $W_{(0)}^{2 n-1}$ we take the resolution of the fiber corresponding to the map of resolutions covering $\pi_{n}: \Omega^{2} S^{2 n+1} \rightarrow S^{2 n-1}$ constructed above. 
The statement regarding the $E_{2}$-term follows from the LES of $E_{2}$-terms, once we check that the map of resolutions induces the homomorphism $v_{0}: \Lambda_{(0)}(2 n+1) \rightarrow$ $\Lambda_{(0)}(2 n-1)$ at least on $E_{2}$. Following the proof of Proposition 2.32 and Lemma 2.29 of [30], let $P_{*}^{\prime}$ be a chain complex of free unstable $A$-modules corresponding to the resolution of $\Omega^{2} S^{2 n+1}$, and $P_{*}^{\prime \prime}$ a chain complex of free unstable $A$-modules for the resolution of $S^{2 n-1}$. Let $\epsilon: P_{*}^{\prime \prime} \rightarrow P_{*}^{\prime}$ denote the difference between the chain map induced by the map of resolutions constructed above, and the given map $v_{0}$. Since $P_{*}^{\prime \prime}$ is acyclic (the resolution of $S^{2 n-1}$ is an Adams resolution), the composite $P_{*}^{\prime \prime} \stackrel{\epsilon}{\rightarrow} P_{*}^{\prime} \stackrel{\sigma}{\rightarrow} P_{*}^{\prime \prime}$ is chain homotopically trivial, where $\sigma$ is double suspension. Thus there is a lifting $P_{*}^{\prime \prime} \rightarrow \operatorname{ker} \sigma$. Now $\operatorname{ker} \sigma$ is a chain complex of free unstable $A$ modules corresponding to a resolution of $W(n)$. This lifting is zero since $\operatorname{ker} \sigma$ is acyclic in the range of dimensions in which $\operatorname{Hom}_{A}\left(P_{*}^{\prime \prime}, Z / p\right)$ is nonzero, which is easy to check by the calculations of section two of [30].

Part 3 ) follows immediately from part 2) by using the resolution of the fiber. Part 2) uses the same argument as Theorem 2.27 and Lemma 2.29 of [30]. Actually no new calculations are needed as the estimates given in [30] yield part 2) directly.

The 4-cell complex at the bottom of $\Omega^{2} W_{(1)}^{2 n p^{2}-1}$ is $V=V(1)$ with the bottom cell in dimension $2 n p^{2}-4$. Checking the $p$-local Freudenthal suspension condition, we see that as long as $n \geq 1$ this $V$ at the bottom is the target of the self map $v_{2}$.

By [16] there is an exponent $k$ such that $v_{2}^{k} \wedge 1$ is the same as $1 \wedge v_{2}^{k}$ as a stable self map of $V \wedge V$. As in [25] we consider the following diagram of pointed mapping spaces. For brevity, denote $\Omega^{2} W_{(1)}^{2 n p^{2}-1}$ by $W, v_{2}^{k}$ by $v$, and set $j=\left|v_{2}^{k}\right|$.

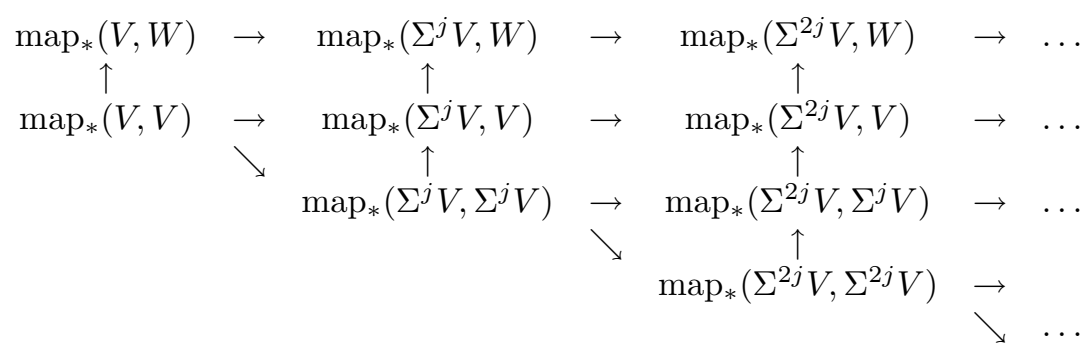

As in [25], this yields a commutative diagram of abelian groups after applying $\pi_{*}$. This produces a homomorphism

$$
\pi_{*}^{\mathrm{S}}(V ; V) \rightarrow v_{2}^{-1} \pi_{*}(W ; V)
$$

which extends to give a homomorphism

$$
v_{2}^{-1} \pi_{*}^{\mathrm{S}}(V ; V) \stackrel{\phi}{\longrightarrow} v_{2}^{-1} \pi_{*}(W ; V) .
$$

Theorem 3.8. The homomorphism $\phi$ is an isomorphism.

Proof. As in [25], we have a corresponding diagram of $E_{2}$-terms

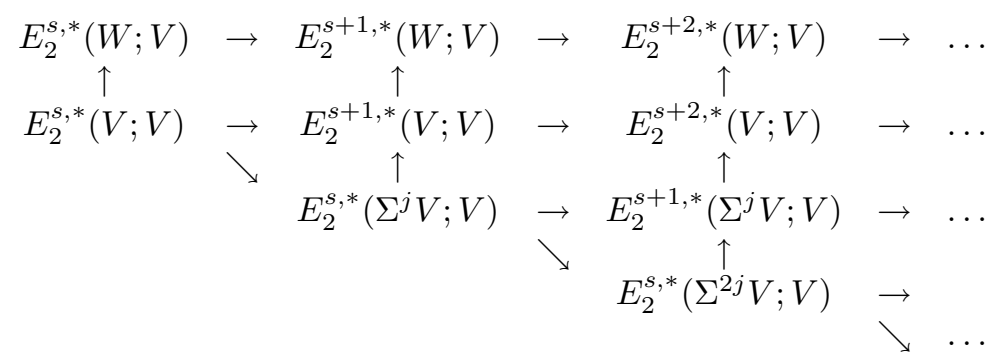


which gives a homomorphism

$$
\operatorname{Ext}_{A}^{s, t}\left(H_{*} V, H_{*} V\right) \rightarrow v_{2}^{-1} E_{2}^{s, t}(W ; V)
$$

which extends to a homomorphism

$$
v_{2}^{-1} \operatorname{Ext}_{A}^{s, t}\left(H_{*} V, H_{*} V\right) \stackrel{\psi}{\rightarrow} v_{2}^{-1} E_{2}^{s, t}(W ; V) .
$$

In [14] it shown that there is an isomorphism

$$
v_{2}^{-1} E_{2}^{s, t}(W) \stackrel{\theta}{\rightarrow} v_{2}^{-1} \operatorname{Ext}_{A}^{s, t}\left(H_{*} V\right) .
$$

Reducing $\bmod V$, we get an isomorphism

$$
v_{2}^{-1} E_{2}^{s, t}(W ; V) \stackrel{\theta}{\rightarrow} v_{2}^{-1} \operatorname{Ext}_{A}^{s, t}\left(H_{*} V, H_{*} V\right) .
$$

Now the argument of [9], Theorem 3.10, shows that the composite

$$
v_{2}^{-1} \operatorname{Ext}_{A}^{s, t}\left(H_{*} V, H_{*} V\right) \stackrel{\psi}{\rightarrow} v_{2}^{-1} E_{2}^{s, t}(W ; V) \stackrel{\theta}{\rightarrow} v_{2}^{-1} \operatorname{Ext}_{A}^{s, t}\left(H_{*} V, H_{*} V\right)
$$

is an isomorphism, and this proves Theorem 3.8.

Returning to the proof of the isomorphism in (3.5), consider the tower of fibrations

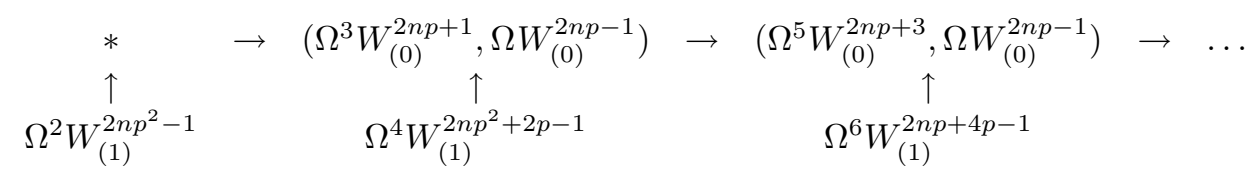

The homotopy colimit of this tower is the pair $\left(Q M^{2 n p-2}, \Omega W_{(0)}^{2 n p-1}\right)$. By applying the functor $v_{2}^{-1} \pi_{*}(\quad ; V)$ to this tower we get a spectral sequence which converges to $v_{2}^{-1} \pi_{*}\left(Q M^{2 n p-2}, \Omega W_{(0)}^{2 n p-1} ; V\right)$.

The complex $C$ has a filtration with subquotients copies of $V$, (see Figure 1), and this filtration is compatible with the above tower. This gives a map of spectral sequences, with $v_{2}$ inverted. Theorem 3.8 says this map of spectral sequences is an isomorphism on $E_{2}$-terms and (3.5) follows.

Now consider (3.2). First note that if we "speed up the filtration" of the pair $\left(Q M^{2 n p-2}, \Omega W_{(0)}^{2 n p-1}\right)$ we get

$$
* \rightarrow\left(\Omega^{2 p+1} W_{(0)}^{2(n+1) p-1}, \Omega W_{(0)}^{2 n p-1}\right) \rightarrow\left(\Omega^{4 p+1} W_{(0)}^{2(n+2) p-1}, \Omega W_{(0)}^{2 n p-1}\right) \rightarrow \ldots
$$

The fiber at each stage is a space $F$ whose bottom $4 p$ cells is a complex $A_{1}$, whose cohomology is $A(1)$, the subalgebra of the Steenrod algebra generated by $\beta$ and $\mathcal{P}^{1}$. Note that $A_{1}$ consists of $p$ copies of $V(1)$ attached together.

In [26] it shown that there is a $v_{2}$-self map $\Sigma^{\left|v_{2}\right|} A_{1} \rightarrow A_{1}$. Again, by the $p$-local Freudenthal suspension condition, this map desuspends to a map of spaces, as long as $A_{1}$ is at least $d-1$-connected, where $d=\frac{2 p^{2}+1}{p-1}+2 p+1$. The dimension of the bottom cell of the first $A_{1}$ is $2 n p^{2}-4$, and so it is the target of the self map $v_{2}$.

Lemma 2.27 of [30] shows that the map

$$
W(n) \stackrel{\Omega \sigma_{1}}{\longrightarrow} \Omega^{2 p} W(n+1)
$$

is covered by a map of resolutions and Lemma 2.29 of [30] shows that the induced map of $E_{2}$-terms is the same as that of the argument above. Thus we have an isomorphism

$$
v_{2}^{-1} E_{2}^{s, t}(F ; V) \stackrel{\theta}{\rightarrow} v_{2}^{-1} \mathrm{Ext}_{A}^{s, t}\left(A_{1}, H_{*} V\right)
$$


and the $v_{2}$-periodic homotopy of $F$ is the stable $v_{2}$-periodic homotopy of $A_{1}$.

Now the proof of (3.2) proceeds exactly as above with $W$ replaced by $F$ and $\operatorname{map}_{*}(V, V)$ replaced by $\operatorname{map}_{*}\left(V, A_{1}\right)$.

\section{REFERENCES}

1. A. K. Bousfield, The localization of spectra with respect to homology, Topology 18 (1979), 257-281. MR 80m:55006

2. $\ldots$ K-localizations and $K$-equivalences of infinite loop spaces, Proc. London Math. Soc. (3) 44 (1982), 291-311. MR 83g:55008

3. Localization and periodicity in unstable homotopy theory, J. Amer. Math. Soc. 7 (1994), no. 4, 831-873. MR 95c:55010

4. J. Caruso, F. Cohen, J. P. May, and L. Taylor, James maps, Segal maps, and the Kahn-Priddy theorem, Trans. Amer. Math. Soc. 218 (1984), 243-283. MR 86g:55007

5. F. Cohen, T. Lada, and J. P. May, The homology of iterated loop spaces, Lecture Notes in Math., vol. 533, Springer-Verlag, 1976. MR 55:9096

6. F. Cohen, J. P. May, and L. R. Taylor, Splitting of certain spaces CX, Math. Proc. Cambridge Philos. Soc. 84 (1978), 465-496. MR 80a:55010

7. F. R. Cohen, J. C. Moore, and J. A. Neisendorfer, Torsion in homotopy groups, Ann. of Math. 109 (1979), 121-168. MR 80e:55024

8. Frederick R. Cohen, The unstable decomposition of $\Omega^{2} \Sigma^{2} X$ and its applications, Math. Z. (1983), 553-568. MR 85d:55008

9. Donald M. Davis and Mark Mahowald, $v_{1}$-periodicity in the unstable Adams spectral sequence, Math. Z. 204 (1990), 319-339. MR 92e:55018

10. E. Dror Farjoun and J. H. Smith, Homotopy localization nearly preserves fibrations, preprint.

11. Brayton Gray, Desuspension at an odd prime, Algebraic Topology, Proceedings, Aarhus 1982, Lecture Notes in Math., vol. 1051, Springer-Verlag, 1984, pp. 360-370. MR 86d:55017

12. __ On the iterated suspension, Topology 27 (1988), no. 3, 301-310. MR 89h:55016

13. $\_$EHP spectra and periodicity. I: Geometric constructions, Trans. Amer. Math. Soc. 340 (1993), no. 2, 595-616. MR 94c:55015

14. — EHP spectra and periodicity. II: $\Lambda$-algebra models, Trans. Amer. Math. Soc. 340 (1993), no. 2, 617-640. MR 94c:55016

15. J. R. Harper and H. R. Miller, On the double suspension homomorphism at odd primes, Trans. Amer. Math. Soc. 273 (1982), no. 1, 319-331. MR 83h:55037

16. M. J. Hopkins and J. H. Smith, Nilpotence and stable homotopy theory, II, to appear in Ann. of Math.

17. D. Kahn, On the stable decomposition of $\Omega^{\infty} \Sigma^{\infty} A$, Lecture Notes in Mathematics, vol. 658, Springer-Verlag, Berlin and New York, 1978, pp. 206-213. MR 80d:55010

18. Nicholas J. Kuhn, The geometry of James-Hopf maps, Pacific J. Math. 102 (1982), no. 2, 397-412. MR 85c:55006

19. _ The transfer and James-Hopf invariants, Math. Z. 196 (1987), 391-405. MR 89d:55033

20. Lisa Langsetmo and Robert D. Thompson, Some applications of $K(1)_{*} W(n)$, The Čech Centennial, Cont. Math., vol. 181, Amer. Math. Soc., 1995, pp. 339-354. MR 95m:55019

21. Mark Mahowald, On the double suspension homomorphism, Trans. Amer. Math. Soc. 214 (1975), 169-178. MR 55:11248

22. 83i: 55019

23. Mark Mahowald and Robert Thompson, A commentary on the 'image of $J$ in the EHP sequence', Algebraic Topology, Proceedings, Seattle 1985, Lecture Notes in Math., vol. 1286, Springer-Verlag, 1987, pp. 298-304. MR 88j:55012

24. MR 92k:55017

25. ㄴ The fiber of the secondary suspension map, Amer. J. Math. 116 (1994), 179-206. MR 95f:55012

26. Binhua Mao, On the realizations of certain $A^{*}$-modules and their properties, Ph.D. thesis, University of Rochester, 1993. 
27. J. E. McClure, The mod $p K$-theory of $Q X, H_{\infty}$-ring spectra and their applications, Lecture Notes in Math., vol. 1176, Springer-Verlag, 1986, pp. 291-377. MR 88e:55001

28. Douglas C. Ravenel, Localization with respect to certain periodic homology theories, Amer. J. Math. 106 (1984), 351-414. MR 85k:55009

29. V. Snaith, A stable decomposition for $\Omega^{n} \Sigma^{n} X$, J. London Math. Soc. 7 (1974), 577-583. MR 49:3918

30. Robert D. Thompson, The $v_{1}$-periodic homotopy groups of an unstable sphere at odd primes, Trans. Amer. Math. Soc. 319 (1990), no. 2, 535-559. MR 90j:55021

Hunter College and the Graduate Center, Cuny, 695 Park Avenue, New York, New YORK 10021

E-mail address: thompson@math.hunter.cuny.edu

$U R L:$ http://math.hunter.cuny.edu/ thompson 